\title{
THE TRANSIENT EUV SKY AS OBSERVED BY ALEXIS
}

\author{
DIANE ROUSSEL-DUPRÉ, JEFFERY J. BLOCH \\ Astrophysics and Radiation Measurements Group, \\ Los Alamos National Laboratory, P.O. Box 1663, MSD436, \\ Los Alamos, NM 87545, USA
}

\section{Introduction}

Los Alamos National Laboratory's ALEXIS satellite, containing six wide field of view EUV telescopes was launched 1993 April 25. ALEXIS is a sky monitor/survey experiment, and with each $50 \mathrm{~s}$ rotation, the satellite's six telescopes (three energy bands 130,178 and $186 \AA$ ) scan nearly half of the sky. Due to the damage sustained at launch by the satellite, the ALEXIS project team has had to spend over a year devising new methods to determine spacecraft attitude knowledge, essential for putting photons back on the sky correctly. These efforts have been successful. A recent review of the ALEXIS point source effort can be found in the Proceedings of IAU Colloquium 152, 1995. This paper reviews the transient systems that have been observed by ALEXIS which fall into two types: cataclysmic variables and unknown, short duration transients.

\section{Transient search}

Three methods are currently being used to detect EUV transients in the ALEXIS data: (i) archival search, (ii) daily automated sky map searches and (iii) manual sky map searches. Real time transient searches were initially done daily by hand. Starting 1995 January 1, these searches were automated, so now the 12,24 and $48 \mathrm{~h}$ sky maps are searched for point sources. Point source locations found in the data by search algorithms are sent via email to the science team for evaluation. Two superoutbursts from VW Hyi, one long outburst from U Gem, an EUV outburst from the AM Her system AR UMa, and fast transients ALEXIS J1139-685 and ALEXIS J1644-032 have been identified as significant transients in the ALEXIS data. 


\section{Fast transients and cataclysmic variable outbursts}

ALEXIS J1139-685 and ALEXIS J1644-032 both have similar time signatures and look to be potentially a new type of transient. Both systems were observed to be in outburst for of order $24 \ldots 36 \mathrm{~h}$. Target of opportunity observations were requested on EUVE for these systems and, in both cases, EUVE was able to slew to target and observe these systems starting less than $24 \ldots 36 \mathrm{~h}$ after maximum light. However in both cases, $24 \mathrm{~h}$ of observations failed to detect the transients.

The ALEXIS VW Hyi observations are the first observations in the EUV with better than one day time resolution. The superoutbursts were observed primarily in the $176 \AA$ telescope but also, weakly in the $130 \AA$ telescope. A pre-outburst short duration enhancement was observed $2.75 \mathrm{~d}$ prior to the 1994 May/June superoutburst optical rise. The EUV superoutburst emission was delayed $0.75 \mathrm{~d}$ from the observed optical rise. Highly variable but persistent EUV emission was only observed for $7.5 \mathrm{~d}$ at the beginning of the outburst. The 1995 May superoutburst was quite different from the previous year. The primary differences are (i) the brightness of this outburst was a factor of two weaker and (ii) although the EUV rise started $1 \mathrm{~d}$ after the optical rise, the maximum flux was observed $4.8 \mathrm{~d}$ after the optical rise.

ALEXIS observed a EUV transient (ALEXIS J1114+43, 1ES 1113+432 or AR UMa) in 1994 late November. This outburst was probably already in progress when the telescope first scanned across it and lasted at least $16 \mathrm{~d}$. The outburst was detected in all three energy channels $(130,178$ and $186 \AA$ ). Ron Remillard obtained optical spectra of AR UMa at the end of the observed outburst confirming the identity as the EUV counterpart.

\section{Summary}

The ALEXIS satellite has been successful in identfying transients in real time. Two major types of transients have been observed: fast transients of duration $24 \ldots 36 \mathrm{~h}$ and cataclysmic variables. Complete monitoring of transient outbursts, which has not been possible with previous pointed, staring satellites, is possible with ALEXIS. This unique capability has resulted in observations of a pre-superoutburst EUV flash in the CV system VW Hyi, as well as significant differences in EUV light curves from superoutburst to superoutburst. In addition, because ALEXIS is a monitoring satellite, it is able to detect CVs that have not been previously identified in the EUV (e.g. AR UMa) thus increasing our knowledge of these systems.

Acknowledgements. These results represent the dedicated efforts of $\mathrm{C}$. Little, M. Kennison, K. Ramsey, S. Ryan, S. Stem, A. Hodapp, B. Edwards and T. Pfafman. This work was supported by the Department of Energy. 